\title{
A Super-Resolution DOA Estimation Method for Fast-Moving Targets in MIMO Radar
}

\author{
Song Liu $\mathbb{D},{ }^{1,2}$ Lan Tang $\mathbb{D},{ }^{1}$ Yechao Bai $\mathbb{D},{ }^{1}$ and Xinggan Zhang ${ }^{1}$ \\ ${ }^{1}$ School of Electronic Science and Engineering, Nanjing University, Nanjing 210023, China \\ ${ }^{2}$ Nanjing Institute of Electronic Technology, Nanjing 210000, China \\ Correspondence should be addressed to Lan Tang; tanglan@nju.edu.cn
}

Received 11 October 2019; Revised 28 December 2019; Accepted 14 January 2020; Published 16 March 2020

Academic Editor: Luis J. Yebra

Copyright $\odot 2020$ Song Liu et al. This is an open access article distributed under the Creative Commons Attribution License, which permits unrestricted use, distribution, and reproduction in any medium, provided the original work is properly cited.

\begin{abstract}
Direction of arrival (DOA) estimation is an essential problem in the radar systems. In this paper, the problem of DOA estimation is addressed in the multiple-input and multiple-output (MIMO) radar system for the fast-moving targets. A virtual aperture is provided by orthogonal waveforms in the MIMO radar to improve the DOA estimation performance. Different from the existing methods, we consider the DOA estimation method with only one snapshot for the fast-moving targets and achieve the superresolution estimation from the snapshot. Based on a least absolute shrinkage and selection operator (LASSO), a denoise method is formulated to obtain a sparse approximation to the received signals, where the sparsity is measured by a new type of atomic norm for the MIMO radar system. However, the denoise problem cannot be solved efficiently. Then, by deriving the dual norm of the new atomic norm, a semidefinite matrix is constructed from the denoise problem to formulate a semidefinite problem with the dual optimization problem. Finally, the DOA is estimated by peak-searching the spatial spectrum. Simulation results show that the proposed method achieves better performance of the DOA estimation in the MIMO radar system with only one snapshot.
\end{abstract}

\section{Introduction}

The orthogonal waveforms are transmitted by multiple-input and multiple-output (MIMO) radar systems [1-6], so the performance of target detection, estimation, and tracking can be improved by the waveform diversity. Generally, the MIMO radar system can be classified into two types: (1) the colocated MIMO radars [7-9], where the distance between antennas is comparable with the wavelength, and the waveform diversity is exploited to improve the target estimation performance with the large virtual aperture; (2) the distributed MIMO radar [10-13], where the spacing between antennas is large, and the different view-angles from the antennas to targets are used to improve the detection performance with the diversity of radar cross-section (RCS). Since the direction of arrival (DOA) estimation problem is addressed in this paper, the colocated MIMO radar is adopted to improve the DOA estimation performance with larger virtual aperture than that in traditional phased array [14].

Many papers have investigated the DOA estimation problem [15-18]. Traditionally, the discrete Fourier transform
(DFT) [19] is used to estimate the DOA, where the received signals are sampled by the antennas in the spatial domain, and then the DOA estimation is equal to a corresponding frequency estimation in the transformed domain. Therefore, the frequency (DOA) in the spatial domain is obtained by the DFT methods, but the resolution of DFT method is limited by Rayleigh criterion. The methods that can break through the Rayleigh criterion are called super-resolution methods. Multiple signal classification (MUSIC) method [20-22], Root-MUSIC [23], and the estimation of signal parameters via rotational invariant techniques (ESPRIT) method [24-26] are three most essential super-resolution methods. The noise subspace and signal subspace are obtained in the MUSIC and ESPRIT methods to estimate the DOA, respectively. A TOD-MUSIC algorithm is proposed in [27] to estimate the DOA in the scenario with low signal-to-noise ratio (SNR) with diversity bistatic MIMO radar.

However, the subspaces are obtained from the estimated covariance matrix of the received signals, so the multiple measurements are needed in MUSIC and ESPRIT methods to achieve a reasonable estimation of the covariance matrix. 
In the MIMO radar system, the multiple measurements are obtained from the multiple pulses, where the measurements are obtained from the output of the pulse compression. In the scenario with fast-moving targets, the multiple measurements are impossible, so the subspace-based methods cannot be used. For the fast-moving targets, the DOA of targets is changing fast and exceeds the limit of the pulse repetition frequency (PRF) [28], so the statistic information, such as the covariance matrix of received signals, cannot be estimated well from the nonstationary pulse signals, and only the single pulse can be adopted to estimate the DOA. Therefore, the fast-moving targets are defined as the ones with nonstationary DOA between adjacent pulses.

The compressed sensing- (CS-) based methods have been proposed $[29,30]$ to improve the DOA estimation performance with fewer measurements, where the target sparsity is exploited in the spatial domain [31-35]. Therefore, the DOA estimation for the sparse targets is transformed into the sparse reconstruction problem in the CS-based methods $[36,37,37]$, and the sparse-based methods can be used in the scenario with fast-moving targets. In [38], a reweighted $\ell_{0}$ norm minimization method with fast iterations is proposed for the DOA estimation in MIMO radar. A fast sparse DOA estimation algorithm for both the white and colored Gaussian noises is proposed in [39] in the scenario with multiple measurement vectors. Xie et al. [40] developed a covariancevector sparsity-aware estimator to estimate the DOA from the MIMO radar.

Usually, the CS-based methods can be classified into the greedy methods and the norm-based methods: (1) in the greedy methods, such as orthogonal matching pursuits (OMP) [41], stagewise OMP (StOMP), and CoSaMP [42], iterations are used to reconstruct the sparse signals; $(2)$ in the norm-based method, the $\ell_{0}$ norm minimization problem is transformed into a $\ell_{1}$ norm minimization problem, which can be solved efficiently with the convex optimization tools. Additionally, sparse Bayesian learning- (SBL-) based methods with the prior assumption of sparse signals are also propped [43], such as SBL method and OGSBI method [44], which can achieve the excellent performance with relatively high computational complexity. Moreover, different from the traditional methods of the sparse reconstruction using the discretized dictionary matrix, the atomic norm-based method as a new type of the norm-based methods realizes the sparse reconstruction in the continuous domain [45-47]. In [48], a semidefinite programming-based method is proposed for the $\ell_{1}$ norm optimization over infinite dictionaries. However, the atomic norm has not studied well in the MIMO radar system for the DOA estimation.

In this paper, the DOA estimation problem in the MIMO radar system is addressed, where a larger virtual aperture is provided by MIMO radar than that in phased array. To estimate the DOA of fast-moving targets, we consider the DOA estimation problem in the scenario with only one measurement from the MIMO radar system. Then, by exploiting both the structure of the MIMO radar system and the target sparsity in the spatial domain, a new type of atomic norm is proposed to obtain the trade-off between the target sparsity and the reconstructed signals. A least absolute shrinkage and selection operator (LASSO) with the novel atomic norm is formulated to denoise the received signals, but the LASSO cannot be solved efficiently, so a semidefinite matrix is constructed theoretically from the dual norm of the new atomic norm. Therefore, a dual optimization problem is obtained and transformed into a semidefinite problem, which can be solved efficiently. With the denoised signals, the DOA is estimated by peak-searching the spatial spectrum.

The remainder of this paper is organized as follows. The MIMO radar system is shown in Section 2. A continue domain super-resolution method for fast-moving targets is proposed in Section 3. Section 4 shows the simulation results, and Section 5 concludes the paper.

Notations. $\|\cdot\|_{1}$, and $\|\cdot\|_{2}$ denote the $\ell_{1}$ norm and the $\ell_{2}$ norm, respectively. $\|\cdot\|^{*}$ denotes the dual norm. $\mathscr{R}\{a\}$ denotes the real part of a complex value. $(\cdot)^{H}$ denotes the Hermitian transpose of a complex matrix/vector.

\section{MIMO Radar System with Fast- Moving Targets}

In this paper, we consider the DOA estimation problem in the MIMO radar with fast-moving targets, and we assume that the number of transmitting antennas is $M$ and that of receiving antenna is $N$. As shown in Figure 1, the orthogonal waveforms are transmitted in the MIMO radar system, and the waveform in the $m$-th transmitting antenna is denoted as $s_{m}(t)(m=0,1, \ldots, M-1)$. Since the orthogonal waveforms are transmitted, we have

$$
\int_{0}^{T} s_{m_{1}}(t) s_{m_{2}}^{\mathrm{H}}(t) \mathrm{d} t= \begin{cases}1, & m_{1}=m_{2} \\ 0, & m_{1} \neq m_{2}\end{cases}
$$

where $T$ is the pulse duration. The MIMO radar system considered in this paper is a type of colocated MIMO radar. Both the transmitting and receiving antennas are equipped in the same system, so the DOA and direction of departure (DOD) are the same. To simplify the analysis, the transmitting and receiving antennas are both uniform linear arrays (ULA). Therefore, in the $n$-th receiving antenna, the received signal can be expressed as

$$
\begin{aligned}
y_{n}(t)= & \sum_{k=0}^{K-1} \sum_{m=0}^{M-1} s_{m}(t) e^{j 2 \pi(m d / \lambda) \sin \left(\theta_{k}\right)} \alpha_{k} e^{j 2 \pi(n d / \lambda) \sin \left(\theta_{k}\right)} \\
& +w_{n}(t)
\end{aligned}
$$

where the number of targets is $K, \alpha_{k}$ denotes the scattering coefficient of the $k$-th target, $d$ is the distance between adjacent antennas, $\lambda$ is the wavelength, $\theta_{k}$ is the DOA of the $k$ th target, and $w_{n}(t)$ is the additive Gaussian noise.

In the $n$-th receiving antenna, the matched filters for orthogonal waveforms are adopted to distinguish the waveforms transmitted by different antennas. For the received signals in the MIMO radar system, if we use the signals directly, the SNR can be lower than $10 \mathrm{~dB}$. However, we can use the pulse compression (i.e., matched filters) to 

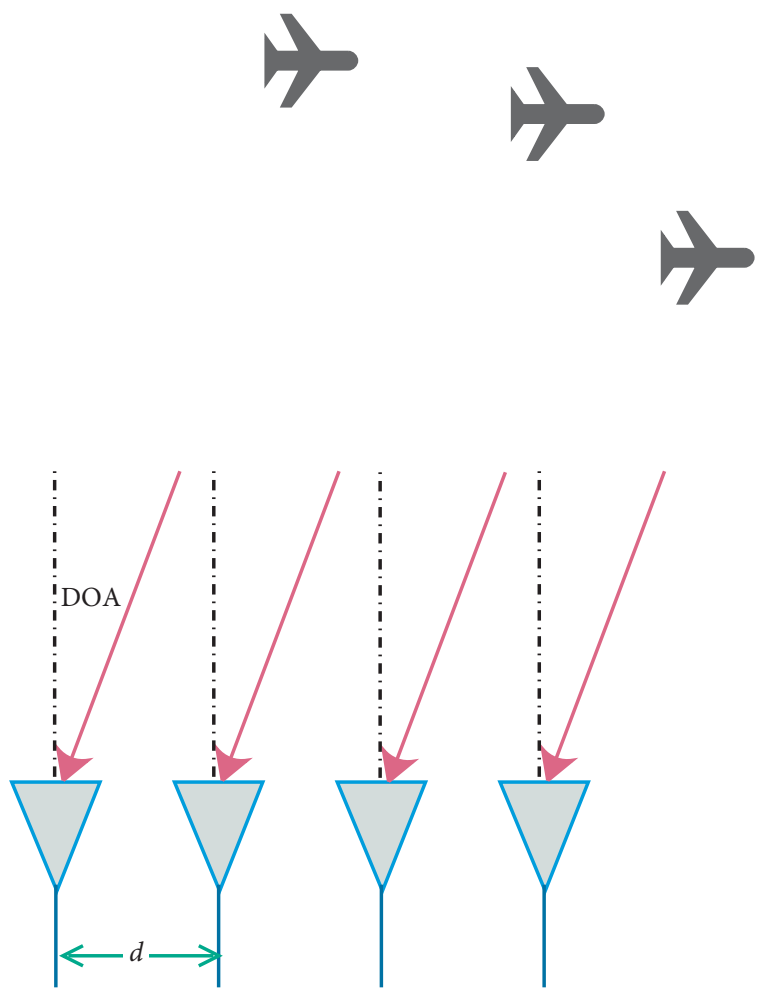

FIgURE 1: The MIMO radar system for DOA estimation.

increase the SNR of the received signals. For the $m$-th waveform $s_{m}(t)$, the corresponding matched filter is $h_{m}(t)=s_{m}^{*}(T-t)$, where $s_{m}^{*}(t)$ is a conjugate function of $s_{m}(t)$. Therefore, in the $n$-th receiving antenna, the signal passing the $m$-th matched filter and sampled at time $T$ can be given as

$$
\begin{aligned}
y_{n, m}= & \sum_{k=0}^{K-1} \int_{0}^{T} s_{m}^{\mathrm{H}}(t) s_{m}(t) e^{j 2 \pi(m d / \lambda) \sin \left(\theta_{k}\right)} \alpha_{k} e^{j 2 \pi(n d / \lambda) \sin \left(\theta_{k}\right)} \mathrm{d} t \\
& +\underbrace{\int_{0}^{T} s_{m}^{\mathrm{H}}(t) w_{n}(t) \mathrm{d} t}_{v_{n, m}} \\
= & \sum_{k=0}^{K-1} \alpha_{k} e^{j 2 \pi((m+n) d / \lambda) \sin \left(\theta_{k}\right)}+v_{n, m} .
\end{aligned}
$$

We define the steering vectors with the direction $\theta$ for the transmitter and receiver, respectively, as follows:

$$
\begin{aligned}
& \mathbf{a}(\theta) \triangleq\left[a_{0}(\theta), a_{1}(\theta), \ldots, a_{M-1}(\theta)\right]^{\mathrm{T}}, \\
& \mathbf{b}(\theta) \triangleq\left[b_{0}(\theta), b_{1}(\theta), \ldots, b_{N-1}(\theta)\right]^{\mathrm{T}},
\end{aligned}
$$

where the $m$-th entry of $\mathbf{a}(\theta)$ is $a_{m}(\theta)=e^{j 2 \pi m d / \lambda \sin (\theta)}$ and the $n$-th entry of $\mathbf{b}(\theta)$ is $b_{n}(\theta)=e^{j 2 \pi n d / \lambda \sin (\theta)}$. Therefore, the received signal passing the matched filter can be simplified as

$$
y_{n, m}=\sum_{k=0}^{K-1} \alpha_{k} a_{m}\left(\theta_{k}\right) b_{n}\left(\theta_{k}\right)+v_{n, m},
$$

where $v_{n, m} \sim \mathscr{C} \mathcal{N}\left(0, \sigma_{W}^{2}\right)$ denotes the additive white Gaussian noise. Then, we can collect all the signals in the $n$-th receiving antenna after the matched filter as follows:

$$
\begin{aligned}
\mathbf{y}_{n} & \triangleq\left[y_{n, 0}, y_{n, 1}, \ldots, y_{n, M-1}\right]^{\mathrm{T}} \\
& =\sum_{k=0}^{K-1} \alpha_{k} b_{n}\left(\theta_{k}\right) \mathbf{a}\left(\theta_{k}\right)+\mathbf{v}_{n},
\end{aligned}
$$

where the noise vector is defined as $\mathbf{v}_{n} \triangleq\left[v_{n, 0}, v_{n, 1}, \ldots\right.$, $\left.v_{n, M-1}\right]^{\mathrm{T}}$. Collect the signals from all the receiving antennas, and we can get the vector with all the information as

$$
\begin{aligned}
\mathbf{y} & \triangleq\left[\mathbf{y}_{0}^{\mathrm{T}}, \mathbf{y}_{1}^{\mathrm{T}}, \ldots, \mathbf{y}_{N-1}^{\mathrm{T}}\right]^{\mathrm{T}} \\
& =\sum_{k=0}^{K-1} \alpha_{k} \mathbf{b}\left(\theta_{k}\right) \otimes \mathbf{a}\left(\theta_{k}\right)+\mathbf{v} \\
& =\mathbf{C} \boldsymbol{a}+\mathbf{v},
\end{aligned}
$$

where we define $\mathbf{v} \triangleq\left[\mathbf{v}_{0}^{\mathrm{T}}, \mathbf{v}_{1}^{\mathrm{T}}, \ldots, \mathbf{v}_{N-1}^{\mathrm{T}}\right]^{\mathrm{T}}$, and $\otimes$ denotes the Kronecker product. Additionally, we define the matrix with steering vectors as

$$
\mathbf{C} \triangleq\left[\mathbf{b}\left(\theta_{0}\right) \otimes \mathbf{a}\left(\theta_{0}\right), \mathbf{b}\left(\theta_{1}\right) \otimes \mathbf{a}\left(\theta_{1}\right), \ldots, \mathbf{b}\left(\theta_{K-1}\right) \otimes \mathbf{a}\left(\theta_{K-1}\right)\right],
$$

and we define the vector for target scattering coefficients as

$$
\alpha \triangleq\left[\alpha_{0}, \alpha_{1}, \ldots, \alpha_{K-1}\right]^{\mathrm{T}} .
$$

Finally, from the system model (7), we try to estimate the DOA $\theta_{k}$ of targets from the received signals $\mathbf{y}$ with only one snapshot. Usually, in the existing methods with super-resolution, the multiple measurements are used, for example, the MUSIC or ESPRIT methods; the multiple measurements are used to estimate the covariance matrix, and the DOAs are estimated from the noise or signal subspaces, which are obtained from the covariance matrix. In this paper, we focus in the scenario with only one measurement, so the proposed DOA estimation method can be used to track the fast-moving targets and can get the target information from just one pulse.

\section{Continue Domain Super-Resolution Estimation Method}

In this section, we will propose a super-resolution method for the DOA estimation in the continuous domain, named as continue domain super-resolution (CDSR) method. To exploit the target sparsity in the spatial domain, we will formulate a system model from (7) by introducing the sparsity. In the traditional methods to exploit the target sparsity, the compressed sensing- (CS-) based methods have been proposed [49], where the dictionary matrix is formulated by discretizing the spatial domain. Then, the DOA information is obtained from the dictionary matrix. However, the off-grid problem will be introduced during discretizing the spatial domain [50,51], where the targets cannot be precise at the discretized angles. Different from the traditional methods, we will propose a sparse-based method in the continuous domain. First, the preliminary for the CDSR will be given as the background knowledge. 
3.1. Preliminary of Dual Norm. For a norm $\|\mathbf{x}\|$ ( $\mathbf{x}$ is a vector with the entries being complex), the dual norm of $\mathbf{x}$ is defined as

$$
\|\mathbf{x}\|^{*}=\sup _{\|\boldsymbol{\rho}\| \leq 1} \mathscr{R}\left\{\boldsymbol{\rho}^{\mathrm{H}} \mathbf{x}\right\} .
$$

Therefore, for the traditional LASSO problem [52], we have

$$
\min _{\mathbf{x}} \frac{1}{2}\|\mathbf{y}-\mathbf{A x}\|_{2}^{2}+\lambda\|\mathbf{x}\|_{1}
$$

where $\mathbf{x}$ is an unknown vector and $\mathbf{y}$ is a known vector. $\mathbf{A}$ is a measurement matrix. The LASSO problem tries to estimate the vector $\mathbf{x}$ from the known vector $\mathbf{y}$ and measurement matrix A. The first term $1 / 2\|\mathbf{y}-\mathbf{A x}\|_{2}^{2}$ describes the accuracy and the second term $\|\mathbf{x}\|_{1}$ is for the reconstruction sparsity. The parameter $\lambda$ is used to control the tradeoff between the reconstruction accuracy and the sparsity of $\mathbf{x}$.

Then, the optimization problem in (11) can be rewritten asas

$$
\begin{array}{ll}
\min _{\mathbf{x}, \mathbf{z}} & \frac{1}{2}\|\mathbf{y}-\mathbf{z}\|_{2}^{2}+\lambda\|\mathbf{x}\|_{1} \\
\text { s.t. } & \mathbf{z}=\mathbf{A x} .
\end{array}
$$

To solve this optimization problem, the corresponding Lagrangian can be expressed asas

$$
\mathscr{L}(\mathbf{x}, \mathbf{z}, \mathbf{l}) \triangleq \frac{1}{2}\|\mathbf{y}-z\|_{2}^{2}+\lambda\|\mathbf{x}\|_{1}+\mathscr{R}\left\{\mathbf{l}^{\mathrm{H}}(\mathbf{z}-\mathbf{A x})\right\},
$$

where $\mathbf{1}$ is the vector of Lagrangian parameter, and $\mathscr{R}\{\cdot\}$ denotes the real part of a complex value. Therefore, the dual optimization problem of (12) can be obtained as

$$
\begin{aligned}
\max _{\mathbf{l}} \min _{\mathbf{x}, \mathbf{z}} \mathscr{L}(\mathbf{x}, \mathbf{z}, \mathbf{l})= & \max _{\mathbf{l}} \min _{\mathbf{x}, \mathbf{z}} \frac{1}{2}\|\mathbf{y}-\mathbf{z}\|_{2}^{2}+\lambda\|\mathbf{x}\|_{1} \\
& +\mathscr{R}\left\{\mathbf{l}^{\mathrm{H}}(\mathbf{z}-\mathbf{A} \mathbf{x})\right\} \\
= & \max _{\mathbf{l}}\left\{\min _{\mathbf{x}} \lambda\|\mathbf{x}\|_{1}-\mathscr{R}\left\{\mathbf{l}^{\mathrm{H}} \mathbf{A} \mathbf{x}\right\}\right. \\
& \left.+\min _{\mathbf{z}} \frac{1}{2}\|\mathbf{y}-\mathbf{z}\|_{2}^{2}+\mathscr{R}\left\{\mathbf{l}^{\mathrm{H}} \mathbf{z}\right\}\right\} .
\end{aligned}
$$

Additionally, we have

$$
\frac{\partial(1 / 2)\|\mathbf{y}-\mathbf{z}\|_{2}^{2}+\mathscr{R}\left\{\mathbf{l}^{\mathrm{H}} \mathbf{z}\right\}}{\partial \mathbf{z}}=\frac{1}{2}(\mathbf{l}-\mathbf{y}+\mathbf{z})^{\mathrm{H}} .
$$

Let $1 / 2(\mathbf{1}-\mathbf{y}+\mathbf{z})^{\mathrm{H}}=0$, and we can obtain

$$
\mathbf{z}=\mathbf{y}-\mathbf{1}
$$

Moreover, from the definition of dual norm, we can obtain [53]

$$
\begin{aligned}
\min _{\mathbf{x}} \lambda\|\mathbf{x}\|_{1}-\mathscr{R}\left\{\mathbf{l}^{\mathrm{H}} \mathbf{A} \mathbf{x}\right\} & =-\lambda \max _{\mathbf{x}}\left\{\mathscr{R}\left\{\frac{1}{\lambda}\left(\mathbf{A}^{\mathrm{H}} \mathbf{l}\right)^{\mathrm{H}} \mathbf{x}\right\}-\|\mathbf{x}\|_{1}\right\} \\
& =I\left(\left\|\mathbf{A}^{\mathrm{H}} \mathbf{l}\right\|_{1}^{*} \leq \lambda\right),
\end{aligned}
$$

where $\|\cdot\|_{1}^{*}$ denotes the dual norm of $\ell_{1}$ norm. $I(\cdot)$ is an indicator function, which is defined as

$$
I(f(x))= \begin{cases}0, & f(x) \text { is true } \\ +\infty, & \text { otherwise. }\end{cases}
$$

Therefore, substitute (16) and (17) in (14), and we can simplify the dual optimization problem as follows:

$$
\max _{\mathbf{l}}\left\{I\left(\left\|\mathbf{A}^{\mathrm{H}} \mathbf{l}\right\|_{1}^{*} \leq \lambda\right)+\frac{1}{2}\|\mathbf{l}\|_{2}^{2}+\mathscr{R}\left\{\mathbf{l}^{\mathrm{H}}(\mathbf{y}-\mathbf{l})\right\}\right\}
$$

which can be rewritten as

$$
\begin{array}{ll}
\underset{\mathbf{l}}{\min } & \|\mathbf{y}-\mathbf{l}\|_{2}^{2} \\
\text { s.t. } & \left\|\mathbf{A}^{\mathrm{H}} \mathbf{l}\right\|_{1}^{*} \leq \lambda .
\end{array}
$$

The original LASSO problem is transformed into the dual optimization problem using the dual norm, which can be solved more efficiently.

3.2. CDSR Method. We will define a new type of atomic norm, which can exploit the target sparsity in the spatial domain and also use the MIMO advantage. The new type of atomic norm [54-57] for MIMO radar is named as MIMO Atomic Norm (AN-MIMO), which is defined as

$\|\mathbf{x}\|_{\mathrm{AN}-\mathrm{MIMO}, 0} \triangleq \inf \left\{P: \mathbf{x}=\sum_{p=0}^{P-1} d_{p} \mathbf{b}\left(\theta_{p}\right) \otimes \mathbf{a}\left(\theta_{p}\right), \theta_{p} \in[0,2 \pi)\right\}$,

where we use the subscript "AN-MIMO, 0" to show the decomposition for the signal $\mathbf{x}$ with minimum number of terms. The AN-MIMO shows that we can use the minimum number of terms formulated by $\mathbf{b}\left(\theta_{p}\right) \otimes \mathbf{a}\left(\theta_{p}\right)$ to describe the signal $\mathbf{x}$. However, as show in (21), we must determine the number of terms, i.e., the parameter $P$, to obtain the decomposition, which is nondeterministic polynomial (NP) hard and cannot be solved easily.

Inspired by the $\ell_{1}$ norm used in CS theory, we can relax the original AN-MIMO by the following equation:

$$
\begin{aligned}
& \|\mathbf{x}\|_{\text {AN-MIMO }} \\
& \triangleq \inf \left\{\sum_{p=0}^{P-1}\left|d_{p}\right|: \mathbf{x}=\sum_{p=0}^{P-1} d_{p} \mathbf{b}\left(\theta_{p}\right) \otimes \mathbf{a}\left(\theta_{p}\right), \theta_{p} \in[0,2 \pi)\right\},
\end{aligned}
$$

where we use the footnote "AN-MIMO" to show that the relaxation equation is different from the original one in (21). The AN-MIMO is described by the sum of absolute values and can be solved by the following semidefinite programming(SDP-) based method. The number of terms $P$ can be obtained by searching the number of peaks in $\left|\mathbf{x}^{H}[\mathbf{b}(\theta) \otimes \mathbf{a}(\theta)]\right|^{2}$.

With the AN-MIMO, the received signal $\mathbf{y}$ can be approximated by a signal $\mathbf{x}$ with the sparse consideration. We can formulate the following optimization (denoising) problem as

$$
\min \frac{1}{2}\|\mathbf{y}-\mathbf{x}\|_{2}^{2}+\lambda\|\mathbf{x}\|_{\mathrm{AN}-\mathrm{MIMO}}
$$

which is a type of LASSO problem, but we use the AN-MIMO to replace the traditional $\ell_{1}$ norm. As shown in (22), the ANMIMO is defined as the sum of the absolute values $\left|d_{p}\right|$, which 
are the coefficients in the atomic decomposition. In the $\ell_{1}$ norm minimization problem with the vector $\mathbf{x}$, the $\ell_{1}$ norm is defined as the absolute sum of the entries in $\mathbf{x}$. Therefore, the ANMIMO norm is similar to the $\ell_{1}$ norm, and both use the sum of the absolute values. Then, the method used in the $\ell_{1}$ norm minimization problem can be introduced in the AN-MIMO problem. Similar to the LASSO problem in Section 3.1, the denoising problem in (24) can be simplified as

$$
\begin{array}{ll}
\min _{\mathbf{x}} & \|\mathbf{y}-\mathbf{x}\|_{2}^{2} \\
\text { s.t. } & \|\mathbf{x}\|_{\text {AN-MIMO }}^{*} \leq \lambda,
\end{array}
$$

where $\|\mathbf{x}\|_{\mathrm{AN}-\mathrm{MIMO}}^{*}$ denotes the dual norm of AN-MIMO $\|\mathbf{x}\|_{\text {AN-MIMO }}$.

Proposition 1. The optimization problem in (23)with the dual norm of AN-MIMO can be solved by the SDP method.

$$
\begin{aligned}
& \min _{\mathbf{x}, \mathbf{F}, \mathfrak{g}}\|\mathbf{y}-\mathbf{x}\|_{2}^{2} \\
& \text { s.t. }\left[\begin{array}{cc}
\mathbf{F} & \mathbf{x} \\
& \\
\mathbf{x}^{H} & g
\end{array}\right] \geqslant 0 \\
& \mathbf{F} \geqslant 0 \\
& 0<g \leq \lambda^{2} \\
& \mathbf{F}=\left[\begin{array}{cccc}
\mathbf{F}_{0,0} & \mathbf{F}_{0,1} & \ldots & \mathbf{F}_{0, N-1} \\
& & & \\
\mathbf{F}_{1,0} & \mathbf{F}_{1,1} & \ldots & \mathbf{F}_{1, N-1} \\
& & & \\
\vdots & \vdots & \ddots & \vdots \\
\mathbf{F}_{N-1,0} & \mathbf{F}_{N-1,1} & \ldots & \mathbf{F}_{N-1, N-1}
\end{array}\right] \\
& \sum_{m_{1}} F_{n_{1}, n_{2}}^{m_{1}, m_{1}+\Delta}= \begin{cases}\frac{1}{N}, & n_{1}=n_{2}, \Delta=0 \\
0, & \text { otherwise }\end{cases}
\end{aligned}
$$

where $\mathbf{x}$ denotes the approximation of received signal $\mathbf{y}$ with the sparse constraint. The matrix $\mathbf{F}$ is a semidefinite matrix and is composed of the block matrix $\mathbf{F}_{n_{1}, n_{2}}$. For the diagonal block matrices $\mathbf{F}_{n_{1}, n_{2}}\left(n_{1}=n_{2}\right)$, the sum of diagonal entries is $1 / N$, and the sum of subdiagonal entries is 0 . However, for the offdiagonal block matrices $\mathbf{F}_{n_{1}, n_{2}}\left(n_{1} \neq n_{2}\right)$, both the sum of the diagonal entries and that of the subdiagonal entries are 0.

In the optimization problem (25), the parameters $\mathbf{x}, \mathbf{F}$, and $g$ are optimized and solved by minimizing $\|\mathbf{y}-\mathbf{x}\|_{2}^{2}$. The optimal $\mathbf{x}$ is denoted as the denoised signal from $\mathbf{y}$. The optimization problem in (25) is a type of SDP problem and can be solved by the interior-point method. According to Section 6.4 in [58], the computation complexity is $\mathcal{O}\left\{(M N)^{3}\right\}$ using the interior-point method to solve the SDP problem (25).

After obtaining the denoised signal $\mathbf{x}$, the DOA can be estimated by the spatial spectrum, which is calculated by $\left|\mathbf{x}^{H}[\mathbf{b}(\theta) \otimes \mathbf{a}(\theta)]\right|^{2}$.

3.3. Proof of Proposition 1. With the definition of dual norm in (10), the dual norm of AN-MIMO can be expressed as

$$
\begin{aligned}
& \|\mathbf{x}\|_{\text {AN-MIMO }}^{*} \\
& =\sup _{\|\bar{x}\|_{\text {AN-MIMO }} \leq 1} \mathscr{R}\left\{\bar{\rho}^{H} x\right\} \\
& \stackrel{(\text { a) }}{=} \sup _{\sum_{p=0}^{P-1}\left|d_{p}\right| \leq 1, \theta_{p} \in[0,2 \pi)} \mathscr{R}\left\{\left(\sum_{p=0}^{P-1} d_{p} \mathbf{b}\left(\theta_{p}\right) \otimes \mathbf{a}\left(\theta_{p}\right)\right)^{\mathrm{H}} \mathbf{x}\right\} \\
& \stackrel{(\text { b) }}{=} \sup _{\left|d^{\prime}\right| \leq 1, \theta^{\prime} \in[0,2 \pi)} \mathscr{R}\left\{\left(d^{\prime} \mathbf{b}\left(\theta^{\prime}\right) \otimes \mathbf{a}\left(\theta^{\prime}\right)\right)^{\mathrm{H}} \mathbf{x}\right\} \\
& \quad \stackrel{(\mathrm{c})}{=} \sup _{\theta \in[0,2 \pi)}\left|(\mathbf{b}(\theta) \otimes \mathbf{a}(\theta))^{H} \mathbf{x}\right| .
\end{aligned}
$$

The equation (a) is obtained from the definition of ANMIMO, and the equation (b) is obtained by choosing the DOA $\theta^{\prime}$ to maximize the correlation between $\mathbf{b}\left(\theta^{\prime}\right) \otimes \mathbf{a}\left(\theta^{\prime}\right)$ and $\mathbf{x}$. The equation (c) is obtained by letting

$$
d^{\prime}=\frac{\mathbf{x}^{\mathrm{H}}\left(\mathbf{b}\left(\theta^{\prime}\right) \otimes \mathbf{a}\left(\theta^{\prime}\right)\right)}{\left|\left(\mathbf{b}\left(\theta^{\prime}\right) \otimes \mathbf{a}\left(\theta^{\prime}\right)\right)^{\mathrm{H}} \mathbf{x}\right|}
$$

Therefore, based on (26), the constraint $\|\mathbf{x}\|_{\text {AN-MIMO }}^{*} \leq \lambda$ in the dual problem (24) can be simplified as

$$
\sup _{\theta \in[0,2 \pi)}\left|(\mathbf{b}(\theta) \otimes \mathbf{a}(\theta))^{\mathrm{H}} x\right| \leq \lambda .
$$

This inequality can be further simplified as a SDP problem. Before getting the SDP problem, we first introduce the Schur complement as follows.

Lemma 2. Given a block matrix $\left[\begin{array}{ll}\mathbf{A} & \mathbf{C} \\ \mathbf{B} & \mathbf{D}\end{array}\right]$, the matrix is a semidefinite matrix if and only if the following conditions are satisfied: the block $\mathbf{A}$ is a semidefinite matrix and $\left(\mathbf{A}-\mathbf{C D}^{-1} \mathbf{B}\right)$ is also a semidefinite matrix.

With the Schur complement, we can formulate a semidefinite matrix $\left[\begin{array}{cc}\mathbf{F} & \mathbf{x} \\ \mathbf{x}^{H} & g\end{array}\right](\mathbf{F} \geqslant 0$ and $g>0)$, then we have

$$
\mathbf{F}-\mathbf{x} \boldsymbol{g}^{-1} \mathbf{x}^{\mathrm{H}} \geqslant 0 \text {. }
$$

Hence, from the definition of semidefinite matrix, we can find that for any vector $\mathbf{u}$, we have $\mathbf{u}^{H}\left(\mathbf{F}-\mathbf{x} g^{-1} \mathbf{x}^{H}\right) \mathbf{u} \geq 0$, which can be rewritten as

$$
\mathbf{u}^{\mathrm{H}} \mathbf{x} \mathbf{x}^{\mathrm{H}} \mathbf{u} \leq g \mathbf{u}^{\mathrm{H}} \mathbf{F u} .
$$

By choosing $\mathbf{u}=\mathbf{b}(\theta) \otimes \mathbf{a}(\theta)$, (30) can be simplified as 
$\left|[\mathbf{b}(\theta) \otimes \mathbf{a}(\theta)]^{\mathrm{H}} \mathbf{x}\right|^{2} \leq g[\mathbf{b}(\theta) \otimes \mathbf{a}(\theta)]^{\mathrm{H}} \mathbf{F}[\mathbf{b}(\theta) \otimes \mathbf{a}(\theta)]$,

where the left side is equal to the square of left side in (28).

Therefore, if we can let $g[\mathbf{b}(\theta) \otimes \mathbf{a}(\theta)]^{\mathrm{H}} \mathbf{F}[\mathbf{b}(\theta) \otimes \mathbf{a}(\theta)] \leq \lambda^{2}$, the constraint (28) can be satisfied. Then, the dual optimization problem (24) can be simplified as

$$
\begin{array}{ll}
\min _{\mathbf{x}, \mathbf{F}, g} & \|\mathbf{y}-\mathbf{x}\|_{2}^{2} \\
\text { s.t. } & {\left[\begin{array}{cc}
\mathbf{F} & \mathbf{x} \\
\mathbf{x}^{\mathrm{H}} & g
\end{array}\right] \geqslant 0} \\
& g>0 \\
& \mathbf{F} \geqslant 0 \\
& g[\mathbf{b}(\theta) \otimes \mathbf{a}(\theta)]^{\mathrm{H}} \mathbf{F}[\mathbf{b}(\theta) \otimes \mathbf{a}(\theta)] \leq \lambda^{2} .
\end{array}
$$

This optimization problem is a type of SDP problem, but cannot be solved directly, so we need to further simplify the expressions. Let $g \leq \lambda^{2}$, and we need to formulate the block matrix $\mathbf{F}$ to satisfy

$$
[\mathbf{b}(\theta) \otimes \mathbf{a}(\theta)]^{\mathrm{H}} \mathbf{F}[\mathbf{b}(\theta) \otimes \mathbf{a}(\theta)] \leq 1 .
$$

We can express the left side as

$$
\begin{aligned}
& {[\mathbf{b}(\theta) \otimes \mathbf{a}(\theta)]^{\mathrm{H}} \mathbf{F}[\mathbf{b}(\theta) \otimes \mathbf{a}(\theta)]=\sum_{m_{1}=0}^{M-1} \sum_{n_{1}=0}^{N-1} \sum_{m_{2}=0}^{M-1} \sum_{n_{2}=0}^{N-1}\left[b_{n_{1}}(\theta) a_{m_{1}}(\theta)\right]^{\mathrm{H}} F_{n_{1} M+m_{1}, n_{2} M+m_{2}}\left[b_{n_{2}}(\theta) a_{m_{2}}(\theta)\right]} \\
& =\sum_{\Delta_{1}=1}^{M-1} \sum_{m_{1}=0}^{M-1-\Delta_{1}} \sum_{n_{1}=0}^{N-1} \sum_{n_{2}=0}^{N-1}\left[b_{n_{1}}(\theta) a_{m_{1}}(\theta)\right]^{\mathrm{H}} F_{n_{1} M+m_{1}, n_{2} M+m_{1}+\Delta_{1}}\left[b_{n_{2}}(\theta) a_{m_{1}+\Delta_{1}}(\theta)\right] \\
& +\sum_{\Delta_{1}=-(M-1)}^{-1} \sum_{m_{1}=-\Delta_{1}}^{M-1} \sum_{n_{1}=0}^{N-1} \sum_{n_{2}=0}^{N-1}\left[b_{n_{1}}(\theta) a_{m_{1}}(\theta)\right]^{\mathrm{H}} F_{n_{1} M+m_{1}, n_{2} M+m_{1}+\Delta_{1}}\left[b_{n_{2}}(\theta) a_{m_{1}+\Delta_{1}}(\theta)\right] \\
& +\sum_{m_{1}=0}^{M-1} \sum_{n_{1}=0}^{N-1} \sum_{n_{2}=0}^{N-1} F_{n_{1} M+m_{1}, n_{2} M+m_{1}} b_{n_{1}}^{\mathrm{H}}(\theta) b_{n_{2}}(\theta) \\
& =\sum_{\Delta_{1}=1}^{M-1} a_{\Delta_{1}}(\theta) \sum_{m_{1}=0}^{M-1-\Delta_{1}}\left\{\sum_{\Delta_{2}=1}^{N-1} b_{\Delta_{2}}(\theta) \sum_{n_{1}=0}^{N-1-\Delta_{2}} F_{n_{1} M+m_{1},\left(n_{1}+\Delta_{2}\right) M+m_{1}+\Delta_{1}}\right. \\
& \left.+\sum_{\Delta_{2}=-(N-1)}^{-1} b_{\Delta_{2}}(\theta) \sum_{n_{1}=-\Delta_{2}}^{N-1} F_{n_{1} M+m_{1},\left(n_{1}+\Delta_{2}\right) M+m_{1}+\Delta_{1}}+\sum_{n_{1}=0}^{N-1} F_{n_{1} M+m_{1}, n_{1} M+m_{1}+\Delta_{1}}\right\} \\
& +\sum_{\Delta_{1}=-(M-1)}^{-1} a_{\Delta_{1}}(\theta) \sum_{m_{1}=-\Delta_{1}}^{M-1}\left\{\sum_{\Delta_{2}=1}^{N-1} b_{\Delta_{2}}(\theta) \sum_{n_{1}=0}^{N-1-\Delta_{2}} F_{n_{1} M+m_{1},\left(n_{1}+\Delta_{2}\right) M+m_{1}+\Delta_{1}}\right. \\
& \left.+\sum_{\Delta_{2}=-(N-1)}^{-1} b_{\Delta_{2}}(\theta) \sum_{n_{1}=-\Delta_{2}}^{N-1} F_{n_{1} M+m_{1},\left(n_{1}+\Delta_{2}\right) M+m_{1}+\Delta_{1}}+\sum \sum_{n_{1}=0}^{N-1} F_{n_{1} M+m_{1}, n_{1} M+m_{1}+\Delta_{1}}\right\} \\
& +\sum_{m_{1}=0}^{M-1}\left\{\sum_{\Delta_{2}=1}^{N-1} b_{\Delta_{2}}(\theta) \sum_{n_{1}=0}^{N-1-\Delta_{2}} F_{n_{1} M+m_{1},\left(n_{1}+\Delta_{2}\right) M+m_{1}}+\sum_{\Delta_{2}=-(N-1)}^{-1} b_{\Delta_{2}}(\theta) \sum_{n_{1}=-\Delta_{2}}^{N-1} F_{n_{1} M+m_{1},\left(n_{1}+\Delta_{2}\right) M+m_{1}}\right. \\
& \left.+\sum_{n_{1}=0}^{N-1} F_{n_{1} M+m_{1}, n_{1} M+m_{1}}\right\} \text {. }
\end{aligned}
$$


Therefore, we can formulate the matrix $\mathbf{F}$ as a following block matrix

$$
\mathbf{F} \triangleq\left[\begin{array}{cccc}
\mathbf{F}_{0,0} & \mathbf{F}_{0,1} & \ldots & \mathbf{F}_{0, N-1} \\
\mathbf{F}_{1,0} & \mathbf{F}_{1,1} & \ldots & \mathbf{F}_{1, N-1} \\
\vdots & \vdots & \ddots & \vdots \\
\mathbf{F}_{N-1,0} & \mathbf{F}_{N-1,1} & \ldots & \mathbf{F}_{N-1, N-1}
\end{array}\right],
$$

where $\mathbf{F}_{n_{1}, n_{2}} \in \mathbb{C}^{M \times M}\left(n_{1}=0,1, \ldots, N-1\right.$ and $n_{2}=0,1, \ldots$, $N-1)$. For all blocks, $\mathbf{F}_{n_{1}, n_{2}}$ has the following characteristics:

$$
\sum_{m_{1}} F_{n_{1}, n_{2}}^{m_{1}, m_{1}+\Delta}= \begin{cases}\frac{1}{N}, & n_{1}=n_{2}, \Delta=0 \\ 0, & \text { otherwise, }\end{cases}
$$

where $F_{n_{1}, n_{2}}^{m_{1}, m_{1}+\Delta}$ denotes the entry of $\mathbf{F}_{n_{1}, n_{2}}$ at the $m_{1}$-th row and $\left(m_{1}+\Delta\right)$-th column. When the characteristics in (36) is satisfied, we can find that the result in (34) is 1 . Therefore, the condition in (33) is also satisfied.

Finally, the optimization problem in (32) is simplified as

$$
\begin{aligned}
& \min _{\mathbf{x}, \mathbf{F}, \mathfrak{g}}\|\mathbf{y}-\mathbf{x}\|_{2}^{2} \\
& \text { s.t. }\left[\begin{array}{cc}
\mathbf{F} & \mathbf{x} \\
& \\
\mathbf{x}^{H} & g
\end{array}\right] \geqslant 0 \\
& \mathbf{F} \geqslant 0 \\
& 0<g \leq \lambda^{2} \\
& \mathbf{F}=\left[\begin{array}{cccc}
\mathbf{F}_{0,0} & \mathbf{F}_{0,1} & \ldots & \mathbf{F}_{0, N-1} \\
& & & \\
\mathbf{F}_{1,0} & \mathbf{F}_{1,1} & \ldots & \mathbf{F}_{1, N-1} \\
\vdots & \vdots & \ddots & \vdots \\
& & & \\
\mathbf{F}_{N-1,0} & \mathbf{F}_{N-1,1} & \ldots & \mathbf{F}_{N-1, N-1}
\end{array}\right] \\
& \sum_{m_{1}} F_{n_{1}, n_{2}}^{m_{1}, m_{1}+\Delta}= \begin{cases}\frac{1}{N}, & n_{1}=n_{2}, \Delta=0 \\
0, & \text { otherwise. }\end{cases}
\end{aligned}
$$

\section{Simulation Results}

We will show the simulation results in this section, where the simulation parameters are shown in Table 1 . We consider the
TABLE 1: Simulation parameters.

\begin{tabular}{lc}
\hline Parameter & Value \\
\hline The SNR of received signals & $20 \mathrm{~dB}$ \\
The number of receiving antennas $N$ & 10 \\
The number of transmitting antennas $M$ & 5 \\
The number of targets $K$ & 3 \\
The space between antennas $d$ & 0.5 wavelength \\
The spatial angles & {$\left[-80^{\circ}, 80^{\circ}\right]$} \\
\hline
\end{tabular}

DOA estimation problem in the scenario with the MIMO radar system, where the transmitter and the receiver are colocated. Without additional explanation, the default parameters are the same with Table 1 . All the simulation results are obtained from a Work Station with 64G Byte RAM and Intel i7 CPU $(3.40 \mathrm{GHz})$. The MATLAB code for the proposed method is available online: https:/drive.google.com/ drive/folders/1_iFF1XEQhKOl7-6Y9uKcy8kK6h6tRbtD? usp $=$ sharing.

In this section, three state-of-art methods are compared with the proposed method:

(i) Direct Method. In this method, the correlation between the received signal $y$ and the steering vector $\mathbf{b}(\theta) \otimes \mathbf{a}(\theta)$ is calculated as $\mathbf{y}^{\mathrm{H}}[\mathbf{b}(\theta) \otimes \mathbf{a}(\theta)]$ to get the spatial spectrum for the DOA estimation.

(ii) OMP Method. Orthogonal matching pursuits (OMP) method [59] is a typical method for sparse reconstruction. Since our method is a sparse-based DOA estimation method, this comparison is needed.

(iii) SBL Method. Sparse Bayesian learning (SBL) method [44] is a Bayesian-based method for the sparse reconstruction. Usually, this method can achieve better reconstruction performance, but with relatively high computational complexity.

First, we show the estimated spatial spectrum for the DOA estimation in Figure 2, where the number of receiving antennas is $N=10$. The estimated DOAs using different methods are given in Table 2 . The parameter $\lambda$ is chosen as $\lambda=2 \sqrt{M N \log M N} \sigma_{n}$. As shown in this figure, the DOAs of targets are, respectively, $-29.474^{\circ}, 0.68532^{\circ}$ and $40.836^{\circ}$. When we use the proposed method, we can get the estimated DOAs as $-29.57^{\circ}, 0.58^{\circ}$ and $41.01^{\circ}$. To describe the DOA estimation performance, we use the root mean square error (RMSE) to measure the errors, and the RMSE is defined as

$$
\mathrm{RMSE} \triangleq \sqrt{\frac{1}{N_{\mathrm{mc}} K} \sum_{n_{\mathrm{mc}}=0}^{N_{\mathrm{mc}}-1}\left\|\hat{\theta}_{n_{\mathrm{mc}}}-\theta\right\|_{2}^{2}}
$$

where $N_{\mathrm{mc}}$ denotes the number of Monte Carlo simulations and $N_{\mathrm{mc}}=10^{3}$ in this paper. $\hat{\theta}_{n_{\mathrm{mc}}}$ is the estimated DOA during the $n_{\mathrm{mc}}$-th Monte Carlo simulation, and $\boldsymbol{\theta}$ is the ground-truth DOA. We can calculate the RMSE (in degree) using the proposed 0.1300 . When the direct method is used to estimate the DOA, the RMSE is 0.4508. The RMSEs of OMP and SBL methods are, respectively, 0.4082 and 0.3985 . 


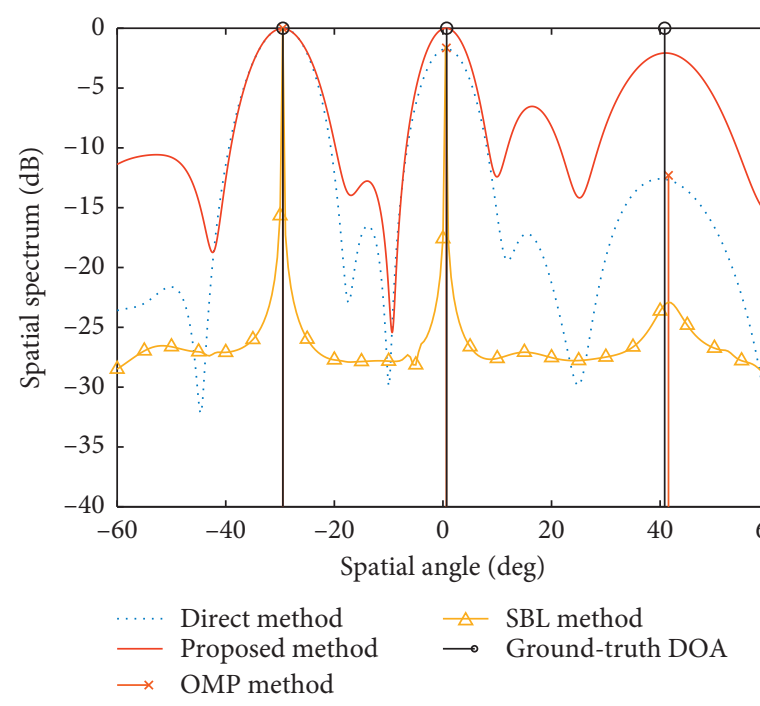

FIgURE 2: The spatial spectrum for DOA estimation using 10 receiving antennas.

TABLE 2: DOA estimation (10 receiving antennas).

\begin{tabular}{lcccc}
\hline Methods & $\begin{array}{c}\text { Target 1 } \\
\left({ }^{\circ}\right)\end{array}$ & $\begin{array}{c}\text { Target 2 } \\
\left({ }^{\circ}\right)\end{array}$ & $\begin{array}{c}\text { Target 3 } \\
\left({ }^{\circ}\right)\end{array}$ & $\begin{array}{c}\text { RMSE } \\
(\mathrm{deg})\end{array}$ \\
\hline Ground-truth DOA & -29.474 & 0.68532 & $40.836^{\circ}$ & - \\
Proposed method & -29.57 & 0.58 & $41.01^{\circ}$ & 0.1300 \\
Direct method & -29.5 & 0.6 & $40.06^{\circ}$ & 0.4508 \\
OMP method & -29.5 & 0.63 & $41.54^{\circ}$ & 0.4082 \\
SBL method & -29.5 & 0.5 & $41.5^{\circ}$ & 0.3985 \\
\hline
\end{tabular}

We can find that the proposed method achieves better DOA estimation performance.

Second, in the scenario with less receiving antennas, we give the simulation results for the DOA estimation in Figure 3, where the number of receiving antennas is $N=4$. As shown in this figure, we can find that the spatial spectrum is worse than the scenario with $N=10$ receiving antennas (Figure 2). The corresponding DOA estimation results are given in Table 3. The RMSE of the proposed method is 0.7009 in degree. The RMSEs of existing methods including the direct method, OMP method, and SBL method are $1.0756,1.0591$, and 0.7623 , respectively. Therefore, in the scenario with less receiving antennas, the proposed method also achieves better performance of the DOA estimation.

Then, Figures 4 and 5 are, respectively, the DOA estimation performance with different SNRs of the received signals, where the number of receiving antennas is $M=10$ in Figure 4 and that in Figure 5 is $M=4$. From these figures, we can find that the proposed method can achieve better performance of DOA estimation in the scenarios with relative high SNR of received signals. Additionally, with $M=10$, the proposed method has better performance at SNR $>25 \mathrm{~dB}$, but the better performance can be achieved at SNR $>15 \mathrm{~dB}$ with $N=4$. Therefore, with more antennas, the performance improvement is significant at higher SNR. The corresponding Cramér-Rao lower bound (CRLB) for the

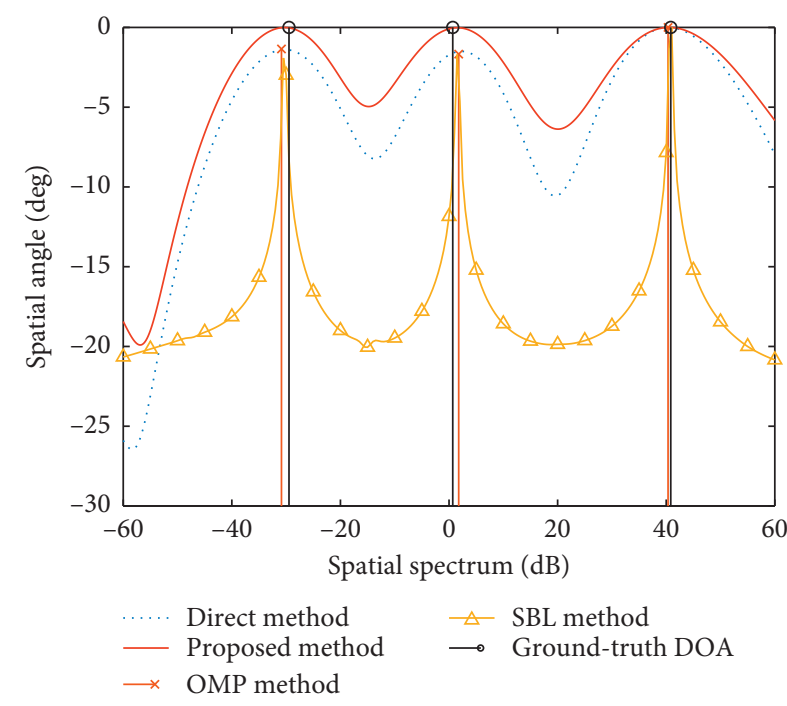

Figure 3: The spatial spectrum for DOA estimation using 4 receiving antennas.

TABLE 3: DOA estimation (4 receiving antennas).

\begin{tabular}{lcccc}
\hline Methods & $\begin{array}{c}\text { Target 1 } \\
\left({ }^{\circ}\right)\end{array}$ & $\begin{array}{c}\text { Target 2 } \\
\left({ }^{\circ}\right)\end{array}$ & $\begin{array}{c}\text { Target 3 } \\
\left({ }^{\circ}\right)\end{array}$ & $\begin{array}{c}\text { RMSE } \\
(\mathrm{deg})\end{array}$ \\
\hline Ground-truth DOA & -29.474 & 0.68532 & 40.836 & - \\
Proposed method & -30.28 & 1.54 & 40.53 & 0.7009 \\
Direct method & -30.28 & 2.29 & 40.34 & 1.0756 \\
OMP method & -30.86 & 1.78 & 40.34 & 1.0591 \\
SBL method & -30.5 & 1.5 & 41 & 0.7623 \\
\hline
\end{tabular}

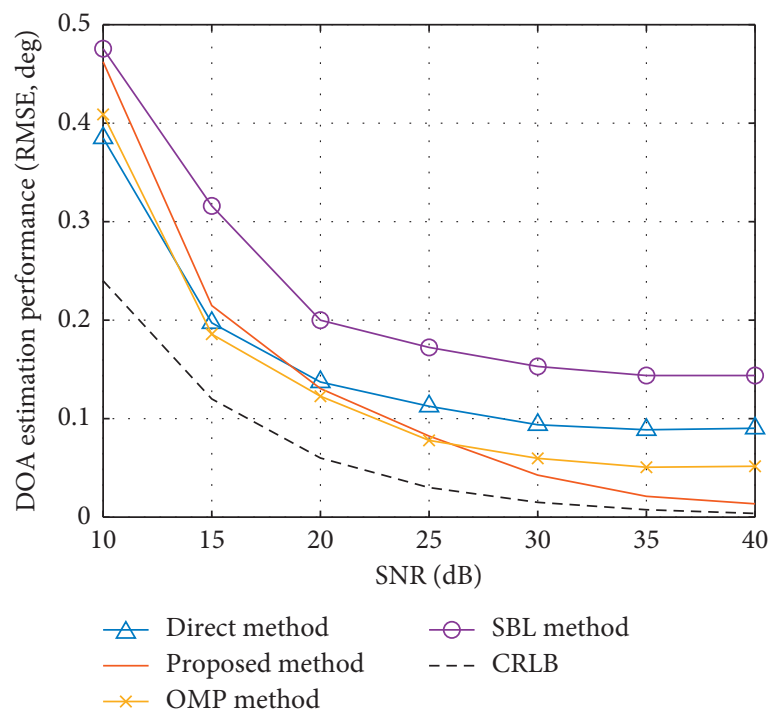

FIgURE 4: The DOA estimation performance with different SNRs (10 receiving antennas).

DOA estimation can be obtained from $[60,61]$. In both Figures 4 and 5, the CRLBs for the DOA estimation are shown. As shown in the figures, the proposed method can 


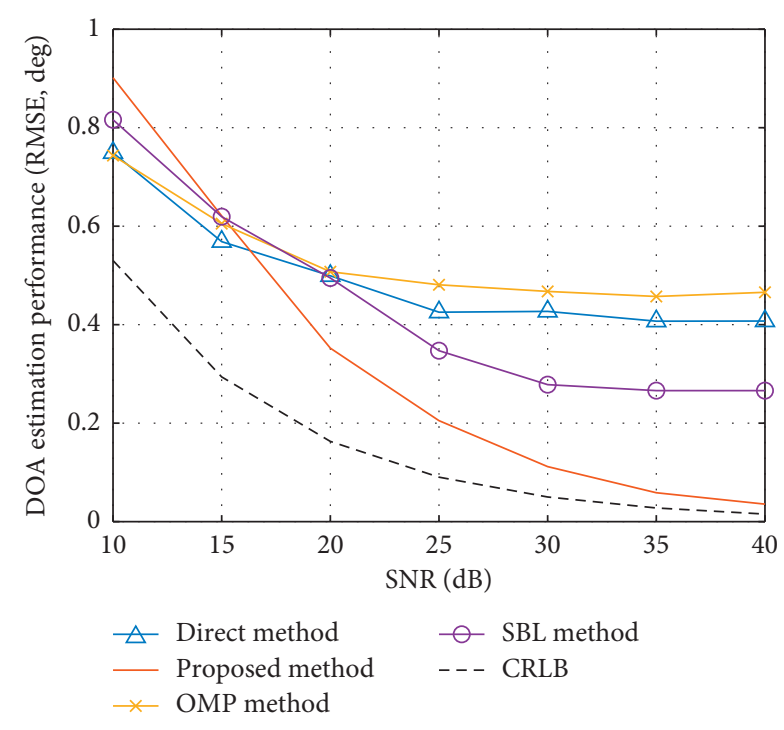

FIGURE 5: The DOA estimation performance with different SNRs (4 receiving antennas).

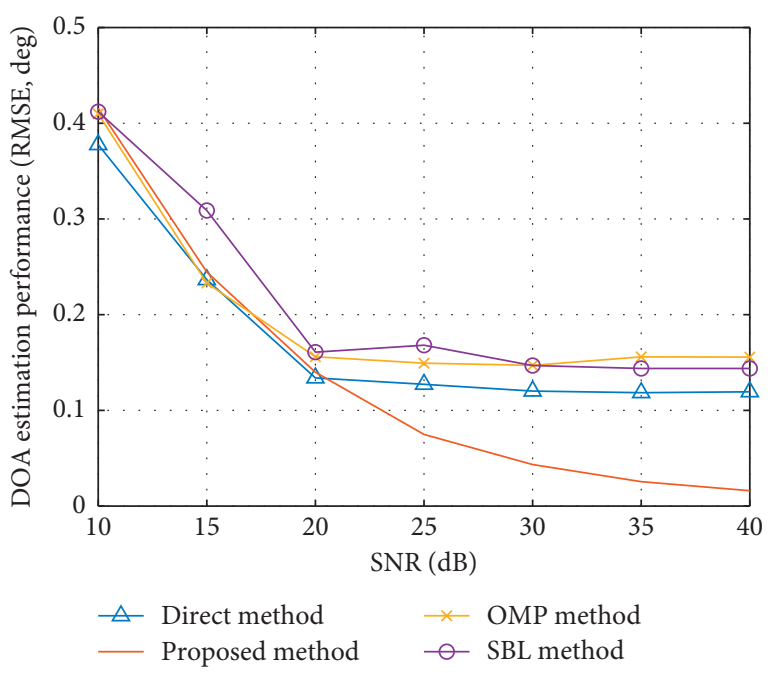

FIGURE 6: The DOA estimation performance with different SNRs (10 transmitting antennas).

approach the CRLB in the scenario with higher SNR $(\mathrm{SNR} \geq 30 \mathrm{~dB})$.

Finally, the DOA estimation performance with more transmitting antennas is shown in Figure 6, where 10 transmitting antennas are adopted. As shown in this figure, we can find that the proposed method achieves better DOA estimation performance at $\mathrm{SNR}>20 \mathrm{~dB}$. Additionally, the performance improvement is more significant in the scenario with higher SNR of the received signals. Compared with Figure 5 using only 5 transmitting antennas, the DOA estimation performance is improved with more antennas. Therefore, the proposed method can achieve better performance of DOA estimation in the scenario with higher SNR, especially using fewer antennas. In Table 4, the computational time is given for the different methods. The computational time of the proposed method is $0.6037 \mathrm{~s}$.
TABle 4: Computational time.

\begin{tabular}{lc}
\hline Methods & Computational time (s) \\
\hline Proposed method & 0.6037 \\
Direct method & 0.02321 \\
OMP method & 0.1051 \\
SBL method & 1.0702 \\
\hline
\end{tabular}

The direct and OMP methods have lower computational time than the proposed method. The SBL method has higher computational complexity with the computational time being $1.0702 \mathrm{~s}$. Therefore, the proposed method can achieve better performance for the DOA estimation with the acceptable computational complexity.

\section{Conclusion}

The DOA estimation problem in the MIMO radar system with fast-moving targets has been addressed, and the new type of atomic norm has been formulated to measure the target sparsity in the spatial domain. The received signals have been denoised by the LASSO-based model. Then, the semidefinite matrix has been constructed to transform the atomic norm minimization problem into the SDP problem, which can be solved efficiently. Simulation results show that the proposed method achieves better DOA estimation performance in the MIMO radar with fast-moving targets. The future work will focus on the radar system optimization to further improve the DOA estimation performance.

\section{Data Availability}

The simulation data used to support the findings of this study are included within the article. Additionally, the Matlab codes for the simulations are available online: https://drive.google. com/drive/folders/1_iFF1XEQhKOl7-6Y9uKcy8kK6h6tRbtD? usp $=$ sharing.

\section{Conflicts of Interest}

The authors declare that they have no conflicts of interest.

\section{Acknowledgments}

This work was supported in part by the National Natural Science Foundation of China (Grant no. 61976113).

\section{References}

[1] J. Liu and J. Li, "Robust detection in MIMO radar with steering vector mismatches," IEEE Transactions on Signal Processing, vol. 67, no. 20, pp. 5270-5280, 2019.

[2] Z. Cao, P. Chen, Z. Chen, and Y. Jin, "DOA estimation for multiple targets in MIMO radar with nonorthogonal signals," Mathematical Problems in Engineering, vol. 2018, Article ID 6465856, 7 pages, 2018.

[3] P. Chen, Z. Cao, Z. Chen, and C. Yu, "Sparse DOD/DOA estimation in a bistatic MIMO radar with mutual coupling effect," Electronics, vol. 7, no. 11, 2018. 
[4] L. Xu, S. Zhou, H. Liu, and J. Liu, "Repeat radar jammer suppression for a colocated MIMO radar," IET Radar, Sonar \& Navigation, vol. 13, no. 9, pp. 1448-1457, 2019.

[5] X. Yu, G. Cui, J. Yang, L. Kong, and J. Li, "Wideband MIMO radar waveform design," IEEE Transactions on Signal Processing, vol. 67, no. 13, pp. 3487-3501, 2019.

[6] P. Chen, Z. Cao, Z. Chen, and X. Wang, "Off-grid DOA estimation using sparse bayesian learning in MIMO radar with unknown mutual coupling," IEEE Transactions on Signal Processing, vol. 67, no. 1, pp. 208-220, 2019.

[7] M. La Manna and D. R. Fuhrmann, "Cramér-rao lower bounds comparison for 2D hybrid-MIMO and MIMO radar," IEEE Journal of Selected Topics in Signal Processing, vol. 11, no. 2, pp. 404-413, 2017.

[8] J. Yan, W. Pu, H. Liu, B. Jiu, and Z. Bao, "Robust chance constrained power allocation scheme for multiple target localization in colocated MIMO radar system," IEEE Transactions on Signal Processing, vol. 66, no. 15, pp. 3946-3957, 2018.

[9] P. Chen, L. Zheng, X. Wang, H. Li, and L. Wu, "Moving target detection using colocated MIMO radar on multiple distributed moving platforms," IEEE Transactions on Signal Processing, vol. 65, no. 17, pp. 4670-4683, 2017.

[10] Y. Du and P. Wei, "An explicit solution for target localization in noncoherent distributed MIMO radar systems," IEEE Signal Processing Letters, vol. 21, no. 9, pp. 1093-1097, 2014.

[11] H. Chen, S. Ta, and B. Sun, "Cooperative game approach to power allocation for target tracking in distributed MIMO radar sensor networks," IEEE Sensors Journal, vol. 15, no. 10, pp. 5423-5432, 2015.

[12] P. Chen and L. Wu, "System optimization for temporal correlated cognitive radar with EBPSK-based MCPC signal," Mathematical Problems in Engineering, vol. 2015, Article ID 302083, 10 pages, 2015.

[13] P. Wang, H. Li, and B. Himed, "A parametric moving target detector for distributed MIMO radar in non-homogeneous environment," IEEE Transactions on Signal Processing, vol. 61, no. 9, pp. 2282-2294, 2013.

[14] R. M. Narayanan, E. T. Gebhardt, and S. P. Broderick, "Through-wall single and multiple target imaging using MIMO radar," Electronics, vol. 6, no. 4, 2017.

[15] M. Ulrich and B. Yang, "Wavelength-diverse MIMO radar: parameter-coupling, array-carrier optimization and direction-of-arrival estimation," IEEE Transactions on Aerospace and Electronic Systems, vol. 55, no. 4, pp. 1920-1932, 2019.

[16] H. Chen, K. Chen, K. Cheng, Q. Chen, Y. Fu, and L. Li, “An efficient hardware accelerator for the MUSIC algorithm," Electronics, vol. 8, no. 5, p. 511, 2019.

[17] S. Liu, W. Wang, L. Fu, and Q. Lu, "Linear prediction-based DOA estimation for directional borehole radar 3-D imaging," IEEE Transactions on Geoscience and Remote Sensing, vol. 57, no. 8, pp. 5493-5501, 2019.

[18] Z. Chen, X. He, Z. Cao, Y. Jin, and J. Li, "Position estimation of automatic-guided vehicle based on MIMO antenna array," Electronics, vol. 7, no. 9, 2018.

[19] B. D. V. Veen and K. M. Buckley, "Beamforming: a versatile approach to spatial filtering," IEEE ASSP Magazine, vol. 5, no. 2, pp. 4-24, 1988.

[20] R. O. Schmidt, "Multiple emitter location and signal parameter estimation," IEEE Transactions on Antennas and Propagation, vol. 34, no. 3, pp. 276-280, 1986.

[21] S. V. Tenneti and P. P. Vaidyanathan, "iMUSIC: a family of MUSIC-like algorithms for integer period estimation," IEEE
Transactions on Signal Processing, vol. 67, no. 2, pp. 367-382, 2019.

[22] M. Wang and A. Nehorai, "Coarrays, MUSIC, and the Cramér-Rao bound," IEEE Transactions on Signal Processing, vol. 65, no. 4, pp. 933-946, 2017.

[23] M. D. Zoltowski, G. M. Kautz, and S. D. Silverstein, "Beamspace root-MUSIC," IEEE Transactions on Signal Processing, vol. 41, no. 1, pp. 344-364, 1993.

[24] R. Roy and T. Kailath, "ESPRIT-estimation of signal parameters via rotational invariance techniques," IEEE Transactions on Acoustics, Speech, and Signal Processing, vol. 37, no. 7, pp. 984-995, 1989.

[25] S. Sahnoun, K. Usevich, and P. Comon, "Multidimensional ESPRIT for damped and undamped signals: algorithm, computations, and perturbation analysis," IEEE Transactions on Signal Processing, vol. 65, no. 22, pp. 5897-5910, 2017.

[26] J. Lin, X. Ma, S. Yan, and C. Hao, "Time-frequency multiinvariance ESPRIT for DOA estimation," IEEE Antennas and Wireless Propagation Letters, vol. 15, pp. 770-773, 2016.

[27] X. Zeng, G. Chen, S. Jiao, S. Fu, and L. Dong, "High-accuracy and low-complexity DOA estimation algorithm for transmitonly diversity bistatic MIMO radar," IEEE Access, vol. 7, pp. 118278-118286, 2019.

[28] J. Yang, C. Liu, and Y. Wang, "Imaging and parameter estimation of fast-moving targets with single-antenna SAR," IEEE Geoscience and Remote Sensing Letters, vol. 11, no. 2, pp. 529-533, 2014.

[29] P. Chen, Z. Cao, Z. Chen, and C. Yu, "Sparse off-grid DOA estimation method with unknown mutual coupling effect," Digital Signal Processing, vol. 90, pp. 1-9, 2019.

[30] L. Liu, X. Zhang, and P. Chen, "Compressed sensing-based DOA estimation with antenna phase errors," Electronics, vol. 8, no. 3, 2019.

[31] S. Uehashi, Y. Ogawa, T. Nishimura, and T. Ohgane, "Prediction of time-varying multi-user MIMO Channels based on DOA estimation using compressed sensing," IEEE Transactions on Vehicular Technology, vol. 68, no. 1, pp. 565-577, 2019.

[32] X. Wu, W.-P. Zhu, and J. Yan, "A toeplitz covariance matrix reconstruction approach for direction-of-arrival estimation," IEEE Transactions on Vehicular Technology, vol. 66, no. 9, pp. 8223-8237, 2017.

[33] P. Zhang, L. Gan, C. Ling, and S. Sun, "Atomic norm denoising-based joint channel estimation and faulty antenna detection for massive MIMO," IEEE Transactions on Vehicular Technology, vol. 67, no. 2, pp. 1389-1403, 2018.

[34] C. Zhou, Y. Gu, X. Fan, Z. Shi, G. Mao, and Y. D. Zhang, "Direction-of-arrival estimation for coprime array via virtual array interpolation," IEEE Transactions on Signal Processing, vol. 66, no. 22, pp. 5956-5971, 2018.

[35] Z. Gao, L. Dai, S. Han, C.-L. I, Z. Wang, and L. Hanzo, "Compressive sensing techniques for next-generation wireless communications," IEEE Wireless Communications, vol. 25, no. 3, pp. 144-153, 2018.

[36] P. Chen, Z. Cao, Z. Chen, L. Liu, and M. Feng, "Compressed sensing-based DOA estimation with unknown mutual coupling effect," Electronics, vol. 7, no. 12, 2018.

[37] X. Zhang, L. Liu, P. Chen, Z. Cao, and Z. Chen, "Gridless sparse direction finding method for correlated signals with gain-phase errors," Electronics, vol. 8, no. 5, 2019.

[38] J. Liu, W. Zhou, F. H. Juwono, and D. Huang, "Reweighted smoothed 10 -norm based DOA estimation for MIMO radar," Signal Processing, vol. 137, pp. 44-51, 2017. 
[39] J. Liu, W. Zhou, and F. H. Juwono, "Joint smoothed $\ell_{0}$-norm DOA estimation algorithm for multiple measurement vectors in MIMO radar," Sensors, vol. 17, no. 5, 2017.

[40] Q. Xie, X. Pan, M. Huang, J. Chen, and S. Xiao, "Sparsitybased direction-of-departure and direction-of-arrival estimation for bistatic multiple-input multiple-output radar," IEEE Access, vol. 7, pp. 118826-118838, 2019.

[41] G. Tan, B. Wu, and T. Herfet, "Performance analysis of OMPbased channel estimations in mobile OFDM systems," IEEE Transactions on Wireless Communications, vol. 17, no. 5, pp. 3459-3473, 2018.

[42] R. G. Baraniuk, T. Goldstein, A. C. Sankaranarayanan, C. Studer, A. Veeraraghavan, and M. B. Wakin, "Compressive video sensing: algorithms, architectures, and applications," IEEE Signal Processing Magazine, vol. 34, no. 1, pp. 52-66, 2017.

[43] L. Wang, L. Zhao, S. Rahardja, and G. Bi, "Alternative to extended block sparse bayesian learning and its relation to pattern-coupled sparse bayesian learning," IEEE Transactions on Signal Processing, vol. 66, no. 10, pp. 2759-2771, 2018.

[44] Z. Yang, L. Xie, and C. Zhang, "Off-grid direction of arrival estimation using sparse bayesian inference," IEEE Trans. Signal Process., vol. 61, no. 1, pp. 38-43, 2012.

[45] S. Beygi, A. Elnakeeb, S. Choudhary, and U. Mitra, "Bilinear matrix factorization methods for time-varying narrowband channel estimation: exploiting sparsity and rank," IEEE Transactions on Signal Processing, vol. 66, no. 22, pp. 60626075, 2018.

[46] D. Yang, G. Tang, and M. B. Wakin, "Super-resolution of complex exponentials from modulations with unknown waveforms," IEEE Transactions on Information Theory, vol. 62, no. 10, pp. 5809-5830, 2016.

[47] Z. Yang, L. Xie, and P. Stoica, "Vandermonde decomposition of multilevel toeplitz matrices with application to multidimensional super-resolution," IEEE Transactions on Information Theory, vol. 62, no. 6, pp. 3685-3701, 2016.

[48] H.-H. Chao and L. Vandenberghe, "Semidefinite representations of gauge functions for structured low-rank matrix decomposition," SIAM Journal on Optimization, vol. 27, pp. 1362-1389, 2016.

[49] Z. Cao, H. Geng, Z. Chen, and P. Chen, "Sparse-based millimeter wave channel estimation with mutual coupling effect," vol. 8, no. 2, p. 358, 2019.

[50] G. Tang, B. N. Bhaskar, P. Shah, and B. Recht, "Compressed sensing off the grid," IEEE Transactions on Information Theory, vol. 59, no. 11, pp. 7465-7490, 2013.

[51] Y. Chi and Y. Chen, "Compressive two-dimensional harmonic retrieval via atomic norm minimization," IEEE Transactions on Signal Processing, vol. 63, no. 4, pp. 1030-1042, 2015.

[52] C.-K. Wen, J. Zhang, K.-K. Wong, J.-C. Chen, and C. Yuen, "On sparse vector recovery performance in structurally orthogonal matrices via LASSO," IEEE Transactions on Signal Processing, vol. 64, no. 17, pp. 4519-4533, 2016.

[53] B. N. Bhaskar, G. Tang, and B. Recht, "Atomic norm denoising with applications to line spectral estimation," IEEE Transactions on Signal Processing, vol. 61, no. 23, pp. 5987-5999, 2013.

[54] H. Jiang, W.-G. Tang, and S.-X. Pang, "Off-grid DOA estimation for nested array using atomic norm minimisation," Electronics Letters, vol. 54, no. 23, pp. 1344-1346, 2018.

[55] W. Tang, H. Jiang, and S. Pang, "Grid-free DOD and DOA estimation for MIMO radar via duality-based $2 \mathrm{D}$ atomic norm minimization,” IEEE Access, vol. 7, pp. 60827-60836, 2019.

[56] Z. Yang, J. Tang, Y. C. Eldar, and L. Xie, "On the sample complexity of multichannel frequency estimation via convex optimization," IEEE Transactions on Information Theory, vol. 65, no. 4, pp. 2302-2315, 2019.

[57] H. Chu, L. Zheng, and X. Wang, "Semi-blind millimeter-wave channel estimation using atomic norm minimization," IEEE Communications Letters, vol. 22, no. 12, pp. 2535-2538, 2018.

[58] Y. Nesterov and A. Nemirovskii, "Interior-point polynomial algorithms in convex programming," Siam, vol. 13, 1994.

[59] J.-F. Determe, J. Louveaux, L. Jacques, and F. Horlin, "On the noise Robustness of simultaneous orthogonal matching pursuit," IEEE Transactions on Signal Processing, vol. 65, no. 4, pp. 864-875, 2017.

[60] Z.-M. Liu, "Conditional Cramér-Rao lower bounds for DOA estimation and array Calibration," IEEE Signal Processing Letters, vol. 21, no. 3, pp. 361-364, 2014.

[61] J. Huang and Q. Wan, "CRLB for DOA estimation in Gaussian and non-Gaussian mixed environments," Wireless Personal Communications, vol. 68, no. 4, pp. 1673-1688, 2013. 